\title{
Protocol
}

\section{An Agar Mount for Observation of Caenorhabditis elegans Embryos}

\author{
Timothy Walston and Jeff Hardin
}

\section{INTRODUCTION}

The Caenorhabditis elegans embryo is particularly amenable to microscopy and embryological studies because of its short developmental time, transparent shell, and nonpigmented cells. The agar mount described in this protocol is an easy way to prepare live C. elegans embryos for microscopic visualization. The mount slightly embeds the embryo in agar to hold it in place. The mount also slightly compresses the embryo to provide consistent orientation such that every embryo will be positioned with either its right side or its left side facing the objective. Other techniques can result in random orientations that complicate analysis and make identification of individual blastomeres more challenging.

\section{RELATED INFORMATION}

Protocols for collection and analysis of four-dimensional (4D) data can be found in Acquisition of 4D DIC Microscopic Data to Determine Cell Contacts in Caenorhabditis elegans Embryos (Walston and Hardin 2010a) and Analysis of 4D DIC Microscopic Data to Determine Cell Contacts in Caenorhabditis elegans Embryos (Walston and Hardin 2010b). An ablation technique for killing individual cells within the embryo (to facilitate the study of cell interactions within the C. elegans embryo) is described in Laser Killing of Blastomeres in Caenorhabditis elegans (Walston and Hardin 2010c). An alternative method for mounting embryos that involves raising a coverslip on grease "feet" is found in Suspended Embryo Mount for Imaging Caenorhabditis elegans (Mohler and Isaacson 2010).

\section{MATERIALS}

CAUTIONS AND RECIPES: Please see Appendices for appropriate handling of materials marked with $<!>$, and recipes for reagents marked with $<\mathbf{R}>$.

\section{Reagents}

Agar (5\%)

Melt the agar just before use.

C. elegans (gravid hermaphrodites)

$<\mathrm{R}>\mathrm{M} 9$ buffer for agar mounts

$<$ R $>$ Valap for agar mounts

Equipment

Aspirator tube assembly (15-in., for mouth pipette)

Bunsen burner

Coverslips, $18 \times 18-\mathrm{mm}(\# 1)$

Eyelash brush (eyelash glued to end of round toothpick)

Microscope

Adapted from Imaging in Developmental Biology (ed. Sharpe and

Wong). CSHL Press, Cold Spring Harbor, NY, USA, 2011 (in press).

Cite as: Cold Spring Harb Protoc; 2010; doi:10.1101/pdb.prot5540

www.cshprotocols.org 
For assembling the mount, a standard stereoscope is required for all steps with C. elegans. To identify early embryos (one to four cells), a total zoom of $80 \mathrm{X}$ or greater is recommended. We use Leica S8 APO microscopes with 10X eyepieces and Leica MZ12.5 microscopes with $16 \mathrm{X}$ eyepieces and 1.0X objective lenses.

Microscope slides, $25 \times 75-\mathrm{mm}$ ( $1 \mathrm{~mm}$ thick) and single depression ( $3 \mathrm{~mm}$ thick)

Paintbrush

Pasteur pipettes (6-in.)

Pick (platinum wire)

To make the pick, insert 1 in. of 30-gauge platinum or $90 \%$ platinum, $10 \%$ iridium wire into a 6 -in Pasteur pipette. Heat it in a flame until the glass melts around the wire. Use flat end hobby pliers or a small tack hammer to flatten the end of the pick.

Pipettes, calibrated glass $(50-\mu \mathrm{L})$

Razor blade

Syringes (1 cc) with 27 gauge $\times$ 1/2-in. needles or scalpel (\#15 curved blade) (see Step 2)

Tape, laboratory label

Tissue (e.g., Kimwipe)

\section{METHOD}

The total time needed for the experienced is 10-15 min and for the novice is $30 \mathrm{~min}$.

1. Use a platinum wire pick to move $\sim 5$ gravid C. elegans hermaphrodites from a culture dish to a single-depression microscope slide mostly filled with M9 buffer for agar mounts.

The number of hermaphrodites needed depends on the number of embryos required at the appropriate stage.

2. By holding a syringe with a needle in each hand, place the needles on either side of a hermaphrodite, and draw the flat sides of the tips of the needles across each other to cut the worm in half transversely (Fig. 1A,B). The embryos will be released from the halves of the hermaphrodite. Use an eyelash brush to carefully prod the halves to expel any remaining embryos.

It is important to cut as close to the vulva as possible to release newly fertilized embryos in the uterus. This step can also be conducted by cutting the worm in half with a \#15 curved blade scalpel.

3. Sort the embryos using the eyelash brush, and brush them together into a group of $\sim 10$ embryos (Fig. 1C).

Embryos will tend to stick slightly to each other when grouped. If you desire a certain stage of embryogenesis, it is at this point that the embryo stage should be assessed and the embryos sorted appropriately. Two-cell stage embryos are the easiest developmental stage to collect.
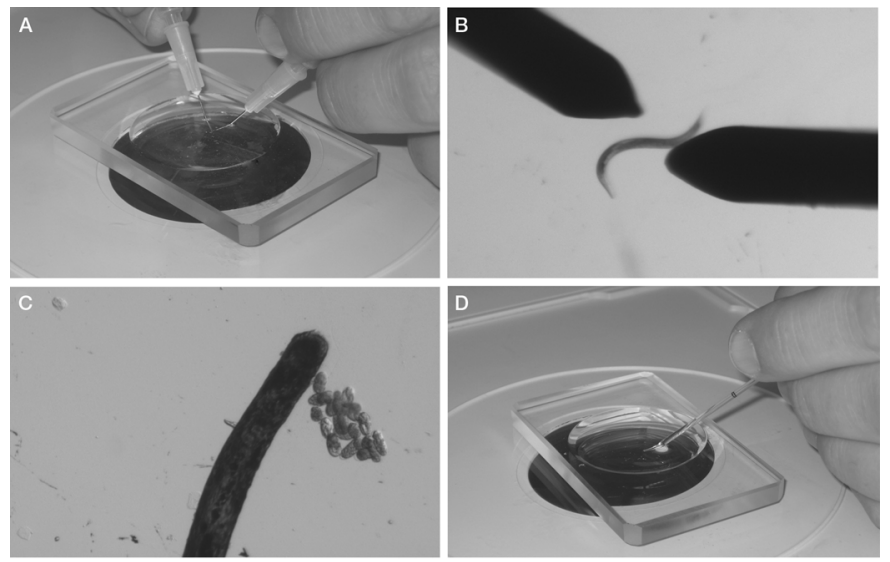

FIGURE 1. Isolation of $C$. elegans embryos and preparation for mounting on a slide. $(A, B)$ Gravid hermaphrodites are cut in half with $27 \times 1 / 2$-in. needles. (C) At a higher magnification, embryos are sorted and are grouped using an eyelash. (D) Embryos and M9 buffer are transferred using a mouth pipette. 
4. Make an agar pad:

i. Using laboratory label tape, tape two flat microscope slides parallel and one slide width apart on the laboratory bench.

ii. Place a third slide between the two taped slides (Fig. 2A).

iii. Using a 6 -in. Pasteur pipette, place three to four drops of molten $5 \%$ agar onto the middle slide.

iv. Immediately lay the fourth slide perpendicular to the other three slides over the agar, and press it over the taped slides to flatten the agar before it cools (Fig. 2B).

5. Once the agar has set, use a razor blade to trim excess agar from the edges of the slides. Carefully slide apart the untaped slides, so that the agar pad is left in the center of one slide.

See Troubleshooting.

6. Prepare a mouth pipette:

i. Heat a glass 50- $\mu \mathrm{L}$ pipette in the flame of a Bunsen burner.

ii. Once the glass is soft and fluid, remove it from the flame, and quickly pull apart the ends.

iii. Break the two ends apart to create a pipette with a tapered end with a diameter of $\sim 40 \mu \mathrm{m}$.

iv. Place the pipette in a mouth pipette aspirator.

7. Using the mouth pipette, transfer the grouping of embryos (from Step 3) and $\sim 20 \mu \mathrm{L}$ of M9 buffer to the corner of the agar pad on the microscope slide (Fig. 1D).

8. Brush the embryos out of the M9 buffer into the center of the slide using the eyelash. Position the embryos in a single layer side by side.

9. Set the edge of a coverslip at the side of the agar pad opposite the M9 buffer and slowly drop it so that the coverslip lands on the embryos before it contacts the M9 buffer. Use a tissue to wick excess buffer from the edges of the coverslip, and wick air bubbles from under the coverslip. See Troubleshooting.

10. Trim excess agar from the edges of the coverslip using a razor blade. Seal the edges of the coverslip with melted valap for agar mounts using a paintbrush (Fig. 2C).

\section{TROUBLESHOOTING}

Problem: The agar pad dries to the slide before it can be used.

[Step 5]

Solution: Make the pad immediately before use. Stereomicroscopes with light sources mounted under the stage have the potential to heat the stage after long use, which can quickly dry agar pads. Using a stereomicroscope with an external bulb or a cool temperature bulb will reduce this problem.

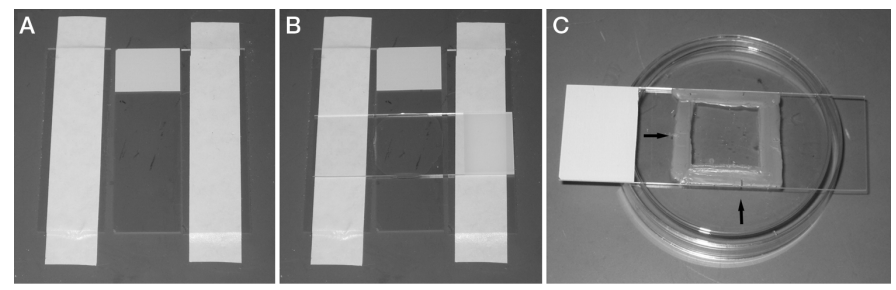

FIGURE 2. Making an agar pad. (A) Three slides are placed on the bench, and the outer two are taped down to the bench. $(B)$ A drop of molten $5 \%$ agar is placed onto the middle slide. A fourth slide is then placed perpendicular to the three original slides. The top slide is compressed over the taped slides. (C) The finished slide is sealed with valap. Using a toothpick to make hash marks in the valap (arrows) aids in finding the grouping of embryos on the compound scope. 
Problem: When a coverslip is placed on the slide, all the embryos wash to the edge of the coverslip. [Step 9]

Solution: Too much M9 buffer is used, and the M9 buffer is hitting the embryos before the coverslip can land on them and hold them in the agar. Use less M9 buffer.

Problem: The slide has air bubbles under the coverslip.

[Step 9]

Solution: Use more M9 buffer. This will allow M9 buffer to completely wash under the coverslip. Too much M9 buffer, however, will cause embryos to wash away (see previous problem).

Problem: Embryos fail to develop after mounting.

Solution: Consider the following:

i. One-cell embryos are especially vulnerable to mechanical stress and are challenging to mount without killing. If studying a later stage of development, the likelihood of embryos surviving is markedly increased if two-cell or later stage embryos are used to make the mount.

ii. Groupings larger than 15-20 embryos often display increased lethality caused by oxygen starvation. By keeping groupings of embryos around 10 embryos, oxygen starvation should not be a problem.

\section{DISCUSSION}

Mounting C. elegans embryos on agar mounts provides a stable long-term environment for microscopic analysis of development. The slight compression from the coverslip will result in embryos reproducibly positioned with either the left or the right side facing toward the objective lens. During later stages of embryogenesis, embryos turn such that left-side views become dorsal views and rightside views become ventral views. Embryos on agar mounts will survive and will hatch from the eggshell on the mount. Embryos prepared with an agar mount are amenable to both light microscopy (with differential interference contrast [DIC] optics) and confocal microscopy.

Alternative methods for mounting embryos, such as poly-L-lysine-coated slides with grease feet (see Suspended Embryo Mount for Imaging Caenorhabditis elegans [Mohler and Isaacson 2010]) or polymer beads, are often used to avoid compression to the embryo. However, these techniques typically result in random embryo orientation, which can complicate analysis of development. Additionally, the slight compression of the coverslip seems to have little to no effect on development; in most cases, avoidance of such compression is unnecessary.

In conclusion, preparing C. elegans embryos on an agar mount is a simple technique that can be easily mastered and is regularly performed by undergraduates in the investigators' laboratories. It provides a consistent embryonic orientation and environment that is suitable for long-term microscopy of C. elegans embryos.

\section{REFERENCES}

Mohler WA, Isaacson AB. 2010. Suspended embryo mount for imaging Caenorhabditis elegans. Cold Spring Harb Protoc doi: 10:1101/pdb.prot5388.

Walston T, Hardin J. 2010a. Acquisition of 4D DIC microscopic data to determine cell contacts in Caenorhabditis elegans embryos. Cold Spring Harb Protoc doi: 10:1101/pdb.prot5541.
Walston T, Hardin J. 2010b. Analysis of 4D DIC microscopic data to determine cell contacts in Caenorhabditis elegans embryos. Cold Spring Harb Protoc doi: 10:1101/pdb.prot5542.

Walston T, Hardin J. 2010c. Laser killing of blastomeres in Caenorhabditis elegans. Cold Spring Harb Protoc doi: 10:1101/ pdb.prot 5543. 


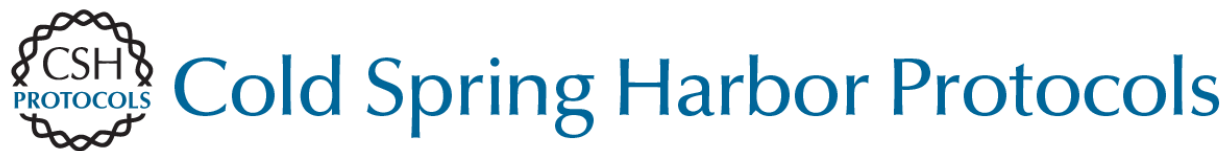

\section{An Agar Mount for Observation of Caenorhabditis elegans Embryos}

Timothy Walston and Jeff Hardin

Cold Spring Harb Protoc; doi: 10.1101/pdb.prot5540

\begin{tabular}{|c|c|}
\hline $\begin{array}{l}\text { Email Alerting } \\
\text { Service }\end{array}$ & Receive free email alerts when new articles cite this article - click here. \\
\hline $\begin{array}{l}\text { Subject } \\
\text { Categories }\end{array}$ & $\begin{array}{l}\text { Browse articles on similar topics from Cold Spring Harbor Protocols. } \\
\text { C. elegans (45 articles) } \\
\text { Cell Biology, general (1382 articles) } \\
\text { Cell Imaging (525 articles) } \\
\text { Developmental Biology (728 articles) } \\
\text { Imaging Development (255 articles) } \\
\text { Imaging/Microscopy, general (579 articles) } \\
\text { In Vivo Imaging (334 articles) } \\
\text { In Vivo Imaging, general (168 articles) } \\
\text { Laboratory Organisms, general (923 articles) } \\
\text { Video Imaging / Time Lapse Imaging (171 articles) } \\
\text { Visualization (524 articles) } \\
\text { Visualization, general (369 articles) }\end{array}$ \\
\hline
\end{tabular}

